\begin{tabular}{|c|l|}
\hline Title & Lightlike surfaces of spacelike curves in de Sitter 3-space \\
\hline Author(s) & FUSHO, Takesi; IZUMIY A, Shyuichi \\
\hline Citation & Hokkaido University Preprint Series in Mathematics, 784, 1-10 \\
\hline Issue Date & 2006-04-14 \\
\hline DOI & 10.14943/83934 \\
\hline Doc URL & http://hdl.handle.net/2115/69592 \\
\hline Type & bulletin (article) \\
\hline File Information & pre784.pdf \\
\hline
\end{tabular}

Instructions for use 


\title{
Lightlike surfaces of spacelike curves in de Sitter 3-space
}

\author{
Takesi FUSHO and Shyuichi IZUMIYA*
}

April 14, 2006

April 14, 2006

\begin{abstract}
The Lorentzian space form with the positive curvature is called de Sitter space which is an important subject in the theory of relativity. In this paper we consider spacelike curves in de Sitter 3-space. We define the notion of lightlike surfaces of spacelike curves in de Sitter 3-space. We investigate the geometric meanings of the singularities of such surfaces.
\end{abstract}

\section{Introduction}

The Lorentzian space form with the positive curvature is called de Sitter space. It is an important subject in the theory of relativity and the astrophysics. De Sitter 4-space is know as a vacuum solution of the Einstein equation. In this paper, we consider, however, spacelike curves in de Sitter 3-space as the most elementary case for the study of higher codimensional spacelike submanifolds in non-flat Lorentzian space forms. For spacelike curves in Minkowski 3-space (i.e., the flat Lorentzian space form) and curves in hyperbolic 3-space have been studied in [4, 5]. In this paper we consider a spacelike curve in de Sitter 3-space and the lightlike surface associated to the curve. The lightlike surface is a line bundle along a spacelike curve whose fibres are lightlike lines which is also characterized that each tangent plane at a regular point is lightlike. The singular point of the lightlike surface corresponds to the point of the spacelike curve which has degenerate contact with a lightcone. We can also consider the contact of spacelike curves with a hyperboloid or an ellipsoid in de Sitter space. However the most interesting case is the study on the contact of spacelike curves with lightcones. Moreover, from the point of view of physics, lightlike surfaces are of importance because they are models of different types of horizons studied in relativity theory $[1,3,7]$. Therefore we stick to lightlike surfaces in this paper. The mail results are Theorems 2.1 and 6.3. These results give a classification

\footnotetext{
${ }^{*}$ Work partially supported by Grant-in-Aid for formation of COE. 'Mathematics of Nonlinear Structure via Singularities'

2000 Mathematics Subject classification. 53A35, 58C27, 58C28

Key Words and Phrases. de Sitter space, lightlike surface, spacelike curves, lightcone
} 
of the singularities of lightlike surfaces for generic spacelike curves in de Sitter 3 -space and a geometric characterization of the singularities. Eventually we find a new Lorentzian invariant $\sigma$ for spacelike curves which describe the contact with ligtcones.

All maps considered here are of class $C^{\infty}$ unless otherwise stated.

\section{Basic concepts and the main result}

We now introduce some basic notions on Minkowski 4-space and spacelike curves. For basic results, see [8]. Let $\mathbb{R}^{4}=\left\{\left(x_{1}, x_{2}, x_{3}, x_{4}\right) \mid x_{1}, x_{2}, x_{3}, x_{4} \in \mathbb{R}\right\}$ be a 4 -dimensional vector space. For any vectors $\boldsymbol{x}=\left(x_{1}, x_{2}, x_{3}, x_{4}\right), \boldsymbol{y}=\left(y_{1}, y_{2}, y_{3}, y_{4}\right)$ in $\mathbb{R}^{4}$, the pseudo scalar product of $\boldsymbol{x}$ and $\boldsymbol{y}$ is defined to be $\langle\boldsymbol{x}, \boldsymbol{y}\rangle=-x_{1} y_{1}+x_{2} y_{2}+x_{3} y_{3}+x_{4} y_{4}$. We call $\left(\mathbb{R}^{4},\langle\rangle,\right)$ a Minkowski 4-space. We write $\mathbb{R}_{1}^{4}$ instead of $\left(\mathbb{R}^{4},\langle\rangle,\right)$. We say that a non-zero vector $\boldsymbol{x} \in \mathbb{R}_{1}^{4}$ is spacelike, lightlike or timelike if $\langle\boldsymbol{x}, \boldsymbol{x}\rangle>0,\langle\boldsymbol{x}, \boldsymbol{x}\rangle=0$ or $\langle\boldsymbol{x}, \boldsymbol{x}\rangle<0$ respectively. We define the signature of a vector $\boldsymbol{x}$ :

$$
\operatorname{sign}(\boldsymbol{x})= \begin{cases}1 & \boldsymbol{x} \text { is spacelike } \\ 0 & \boldsymbol{x} \text { is lightlike } \\ -1 & \boldsymbol{x} \text { is timelike }\end{cases}
$$

The norm of the vector $\boldsymbol{x} \in \mathbb{R}_{1}^{4}$ is defined by $\|\boldsymbol{x}\|=\sqrt{|\langle\boldsymbol{x}, \boldsymbol{x}\rangle|}$. For a vector $\boldsymbol{v} \in \mathbb{R}_{1}^{4}$ and a real number $c$, we define a hyperplane with pseudo normal $\boldsymbol{v}$ by $H P(\boldsymbol{v}, c)=\left\{\boldsymbol{x} \in \mathbb{R}_{1}^{4} \mid\langle\boldsymbol{x}, \boldsymbol{v}\rangle=c\right\}$. We call $H P(\boldsymbol{v}, c)$ a spacelike hyperplane, a timelike hyperplane or a lightlike hyperplane if $\boldsymbol{v}$ is timelike, spacelike or lightlike respectively. We also define de Sitter 3-space by

$$
S_{1}^{3}=\left\{\boldsymbol{x} \in \mathbb{R}_{1}^{4} \mid\langle\boldsymbol{x}, \boldsymbol{x}\rangle=1\right\} .
$$

For any $\boldsymbol{x}=\left(x_{1}, x_{2} . x_{3}, x_{4}\right), \boldsymbol{y}=\left(y_{1}, y_{2}, y_{3}, y_{4}\right), \boldsymbol{z}=\left(z_{1}, z_{2}, z_{3}, z_{4}\right) \in \mathbb{R}^{4}$,we define a vector

$$
\boldsymbol{x} \wedge \boldsymbol{y} \wedge \boldsymbol{z}=\left|\begin{array}{cccc}
-\boldsymbol{e}_{1} & \boldsymbol{e}_{2} & \boldsymbol{e}_{3} & \boldsymbol{e}_{4} \\
x_{1} & x_{2} & x_{3} & x_{4} \\
y_{1} & y_{2} & y_{3} & y_{4} \\
z_{1} & z_{2} & z_{3} & z_{4}
\end{array}\right|
$$

where $\left(\boldsymbol{e}_{1}, \boldsymbol{e}_{2}, \boldsymbol{e}_{3}, \boldsymbol{e}_{4}\right)$ is the canonical basis of $\mathbb{R}_{1}^{4}$ and $\boldsymbol{x}_{i}=\left(x_{1}^{i}, x_{2}^{i}, x_{3}^{i}, x_{4}^{i}\right)$. We can easily show that $\langle\boldsymbol{a},(\boldsymbol{x} \wedge \boldsymbol{y} \wedge \boldsymbol{z})\rangle=\operatorname{det}(\boldsymbol{a}, \boldsymbol{x}, \boldsymbol{y}, \boldsymbol{z})$.

We now describe the explicit differential geometry on spacelike curves in $S_{1}^{3}$. Let $\gamma: I \longrightarrow S_{1}^{3}$ be a regular curve. We say that a regular curve $\gamma$ is spacelike if $\dot{\gamma}(t)$ is spacelike at any $t \in I$, where $\dot{\gamma}=d \boldsymbol{\gamma} / d t$. Since $\gamma$ is a spacelike regular curve, we can reparametrise $\gamma$ by the arclength $s=s(t)$. Hence, we may assume that $\gamma(s)$ is a unit speed curve. So we have the tangent vector $\boldsymbol{t}(s)=\boldsymbol{\gamma}^{\prime}(s)$ with $\|\boldsymbol{t}(s)\|=1$. In the case when $\left\langle\boldsymbol{t}^{\prime}(s), \boldsymbol{t}^{\prime}(s)\right\rangle \neq 1$, we have a unit vector $\boldsymbol{n}(s)=\frac{\boldsymbol{t}^{\prime}(s)-\gamma(s)}{\left\|\boldsymbol{t}^{\prime}(s)-\gamma(s)\right\|}$. Moreover, define $\boldsymbol{e}(s)=\gamma(s) \wedge \boldsymbol{t}(s) \wedge \boldsymbol{n}(s)$, then we have a pseudo orthonormal frame $\{\boldsymbol{\gamma}(s), \boldsymbol{t}(s), \boldsymbol{n}(s), \boldsymbol{e}(s)\}$ of $\mathbb{R}_{1}^{4}$ along $\boldsymbol{\gamma}$. By the standard arguments, we can 
show the following Frenet-Serret type formulae: Under the assumption that $\left\langle\boldsymbol{t}^{\prime}(s), \boldsymbol{t}^{\prime}(s)\right\rangle \neq 1$,

$$
\left\{\begin{array}{l}
\boldsymbol{\gamma}^{\prime}(s)=\boldsymbol{t}(s) \\
\boldsymbol{t}^{\prime}(s)=-\gamma(s)+\kappa_{g}(s) \boldsymbol{n}(s) \\
\boldsymbol{n}^{\prime}(s)=\kappa_{g}(s) \delta(\gamma(s)) \boldsymbol{t}(s)+\tau_{g}(s) \boldsymbol{e}(s) \\
\boldsymbol{e}^{\prime}(s)=\tau_{g}(s) \boldsymbol{n}(s)
\end{array}\right.
$$

where $\delta(\gamma(s))=-\operatorname{sign}(\boldsymbol{n}(s)), \kappa_{g}(s)=\left\|\boldsymbol{t}^{\prime}(s)+\boldsymbol{\gamma}(s)\right\|$ and

$$
\tau_{g}(s)=-\frac{\delta(\gamma(s))}{\kappa_{g}^{2}(s)} \operatorname{det}\left(\gamma(s), \gamma^{\prime}(s), \gamma^{\prime \prime}(s), \gamma^{\prime \prime \prime}(s)\right)
$$

We now define a surface in de Sitter 3 -space associated to a spacelike curve. Let $\gamma: I \longrightarrow S_{1}^{3}$ be a unit speed spacelike curve. We define $L S_{\gamma}: I \times \mathbb{R} \longrightarrow S_{1}^{3}$ by

$$
L S_{\gamma}^{ \pm}(s, u)=\gamma(s)+u(\boldsymbol{n}(s) \pm \boldsymbol{e}(s))
$$

We call $L S_{\gamma}^{ \pm}(s, u)$ the lightlike surface of $\boldsymbol{\gamma}$. We also define the following model surfaces: For any $\boldsymbol{v}_{0} \in S_{1}^{3}, L C^{2}\left(\boldsymbol{v}_{0}\right)=\left\{\boldsymbol{u} \in S_{1}^{3} \mid\left\langle\boldsymbol{u}, \boldsymbol{v}_{0}\right\rangle-1=0\right\}$ is called a lightcone. The main purpose in this paper is to study the Lorentzian geometric meanings of the singularities of the lightlike surface. We define a new invariant $\sigma$ of a spacelike curve in $S_{1}^{3}$ by

$$
\sigma(s)=\kappa_{g}{ }^{\prime}(s) \mp \kappa_{g}(s) \tau_{g}(s) .
$$

On the other hand, Let $F: S_{1}^{3} \longrightarrow \mathbb{R}$ be a submersion and $\gamma: I \longrightarrow S_{1}^{3}$ be a spacelike curve. We say that $\gamma$ and $F^{-1}(0)$ have $k$-point contact for $t=t_{0}$ if the function $g(t)=F \circ \gamma(t)$ satisfies $g\left(t_{0}\right)=g^{\prime}\left(t_{0}\right)=\cdots=g^{(k-1)}\left(t_{0}\right)=0, g^{(k)}\left(t_{0}\right) \neq 0$. We also say that $\gamma$ and $F^{-1}(0)$ have at least $k$-point contact for $t=t_{0}$ if the function $g(t)=F \circ \gamma(t)$ satisfies $g\left(t_{0}\right)=g^{\prime}\left(t_{0}\right)=\cdots=$ $g^{(k-1)}\left(t_{0}\right)=0$. The main result in this paper is as follows.

Theorem 2.1 Let $\boldsymbol{\gamma}: I \longrightarrow S_{1}^{3}$ be a unit speed spacelike curve with $\left\langle\boldsymbol{t}^{\prime}(s), \boldsymbol{t}^{\prime}(s)\right\rangle \neq 1$. For $\boldsymbol{v}_{0}=L S_{\gamma}^{ \pm}\left(s_{0}, u_{0}\right)$ and the lightcone $L C^{2}\left(\boldsymbol{v}_{0}\right)=\left\{\boldsymbol{u} \in S_{1}^{3} \mid\left\langle\boldsymbol{u}, \boldsymbol{v}_{0}\right\rangle-1=0\right\}$, we have the followings:

(1) $\boldsymbol{\gamma}$ and $L C^{2}\left(\boldsymbol{v}_{0}\right)$ have at least 2-point contact for $s_{0}$.

(2) $\boldsymbol{\gamma}$ and $L C^{2}\left(\boldsymbol{v}_{0}\right)$ have 3-point contact for $s_{0}$ if and only if

$$
\boldsymbol{v}_{0}=\boldsymbol{\gamma}\left(s_{0}\right)-\frac{1}{\delta\left(\boldsymbol{\gamma}\left(s_{0}\right)\right) \kappa_{g}\left(s_{0}\right)}\left(\boldsymbol{n}\left(s_{0}\right) \pm \boldsymbol{e}\left(s_{0}\right)\right) \text { and } \sigma\left(s_{0}\right) \neq 0 .
$$

Under this condition, the germ of Image $L S_{\gamma}^{ \pm}$at $L S_{\gamma}^{ \pm}\left(s_{0}, u_{0}\right)$ is diffeomorphic to the cuspidal edge $C \times \mathbb{R}$.

(3) $\boldsymbol{\gamma}$ and $L C^{2}\left(\boldsymbol{v}_{0}\right)$ have 4-point contact for $s_{0}$ if and only if

$$
\boldsymbol{v}_{0}=\gamma\left(s_{0}\right)-\frac{1}{\delta\left(\boldsymbol{\gamma}\left(s_{0}\right)\right) \kappa_{g}\left(s_{0}\right)}\left(\boldsymbol{n}\left(s_{0}\right) \pm \boldsymbol{e}\left(s_{0}\right)\right), \sigma\left(s_{0}\right)=0 \text { and } \sigma^{\prime}\left(s_{0}\right) \neq 0
$$

Under this condition, the germ of Image $L S_{\gamma}^{ \pm}$at $L S_{\gamma}^{ \pm}\left(s_{0}, u_{0}\right)$ is diffeomorphic to the swallowtail $S W$. 
Here, $C \times \mathbb{R}=\left\{\left(x_{1}, x_{2}\right) \mid x_{1}{ }^{2}=x_{2}{ }^{3}\right\} \times \mathbb{R}$ is the cuspidal edge and

$$
S W=\left\{\left(x_{1}, x_{2}, x_{3}\right) \mid x_{1}=3 u^{4}+u^{2} v, x_{2}=4 u^{3}+2 u v, x_{3}=v\right\}
$$

is the swallowtail (cf., Fig. 1).

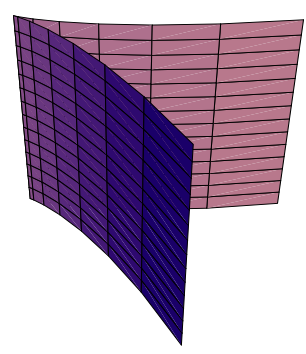

cuspdialedge

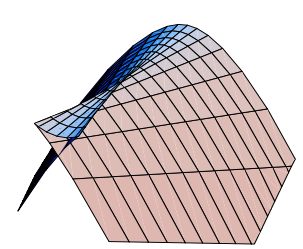

swallowtail

Fig.1.

We remark that the above theorem gives a classification of the singularities of the lightlike surface of a generic spacelike curve in de Sitter 3-space (cf., Theorem 6.3).

\section{$3 \quad$ Extended spacelike height functions}

In this section we consider a family of Lorentzian invariant functions on a spacelike curve in de Sitter 3-space in order to describe the lightlike surface of the spacelike curve. Let $\gamma: I \longrightarrow S_{1}^{3}$ be a unit speed spacelike curve with $\left\langle\boldsymbol{t}^{\prime}(s), \boldsymbol{t}^{\prime}(s)\right\rangle \neq 1$. We now define a family of functions

$$
\bar{H}: I \times S_{1}^{3} \longrightarrow \mathbb{R}
$$

by $\bar{H}(s, \boldsymbol{v})=\langle\boldsymbol{\gamma}(s), \boldsymbol{v}\rangle-1$. We call $\bar{H}$ a extended spacelike height function of $\boldsymbol{\gamma}$. We denote that $\bar{h}_{v}(s)=\bar{H}(s, \boldsymbol{v})$.

Proposition 3.1 For a unit speed spacelike curve $\boldsymbol{\gamma}: I \longrightarrow S_{1}^{3}$ with $\left\langle\boldsymbol{t}^{\prime}(s), \boldsymbol{t}^{\prime}(s)\right\rangle \neq 1$, we have the followings:

(1) $\bar{h}_{v}(s)=0$ if and only if there exist $\lambda, \mu, \nu \in \mathbb{R}$ with $\lambda^{2}-\delta(\gamma(s)) \mu^{2}+\delta(\gamma(s)) \nu^{2}=0$ such that $\boldsymbol{v}=\boldsymbol{\gamma}(s)+\lambda \boldsymbol{t}(s)+\boldsymbol{\mu n}(s)+\nu \boldsymbol{e}(s)$.

(2) $\bar{h}_{v}(s)=\bar{h}_{v}^{\prime}(s)=0$ if and only if if there exists $\mu \in \mathbb{R}$ such that $\boldsymbol{v}=\gamma(s)+\mu \boldsymbol{n}(s) \pm \mu \boldsymbol{e}(s)$.

(3) $\bar{h}_{v}(s)=\bar{h}_{v}^{\prime}(s)=\bar{h}_{v}^{\prime \prime}(s)=0$ if and only if if

$$
\boldsymbol{v}=\boldsymbol{\gamma}(s)-\frac{1}{\delta(\boldsymbol{\gamma}(s)) \kappa_{g}(s)} \boldsymbol{n}(s) \mp \frac{1}{\delta(\boldsymbol{\gamma}(s)) \kappa_{g}(s)} \boldsymbol{e}(s) .
$$

(4) $\bar{h}_{v}(s)=\bar{h}_{v}^{\prime}(s)=\bar{h}_{v}^{\prime \prime}(s)=\bar{h}_{v}^{\prime \prime \prime}(s)=0$ if and only if if

$$
\boldsymbol{v}=\boldsymbol{\gamma}(s)-\frac{1}{\delta(\boldsymbol{\gamma}(s)) \kappa_{g}(s)} \boldsymbol{n}(s) \mp \frac{1}{\delta(\boldsymbol{\gamma}(s)) \kappa_{g}(s)} \boldsymbol{e}(s) \text { and } \sigma(s)=0 \text {. }
$$

(5) $\bar{h}_{v}(s)=\bar{h}_{v}^{\prime}(s)=\bar{h}_{v}^{\prime \prime}(s)=\bar{h}_{v}^{\prime \prime \prime}(s)=\bar{h}_{v}^{(4)}(s)=0$ if and only if if

$$
\boldsymbol{v}=\gamma(s)-\frac{1}{\delta(\gamma(s)) \kappa_{g}(s)} \boldsymbol{n}(s) \mp \frac{1}{\delta(\gamma(s)) \kappa_{g}(s)} \boldsymbol{e}(s), \sigma(s)=0 \text { and } \sigma^{\prime}(s)=0 .
$$


Proof. (1) Since $\boldsymbol{v} \in S_{1}^{3}$, there exist $\omega, \lambda, \mu, \nu \in \mathbb{R}$ with $\omega^{2}+\lambda^{2}-\delta(\gamma(s)) \mu^{2}+\delta(\gamma(s)) \nu^{2}=1$ such that $\boldsymbol{v}=\lambda \boldsymbol{t}(s)+\mu \boldsymbol{n}(s)+\nu \boldsymbol{e}(s)$. It follows $\bar{h}_{v}(s)=\langle\boldsymbol{\gamma}(s), \boldsymbol{v}\rangle-1=0$ that we have $\omega=1$, so that $\boldsymbol{v}=\gamma(s)+\lambda \boldsymbol{t}(s)+\mu \boldsymbol{n}(s)+\nu \boldsymbol{e}(s)$. The converse direction also holds.

(2) Since $\bar{h}_{v}^{\prime}(s)=\langle\boldsymbol{t}(s), \boldsymbol{v}\rangle=0$, we have $\lambda=0$. It follows from the fact $-\delta(\gamma(s)) \mu^{2}+$ $\delta(\gamma(s)) \nu^{2}=0$ that $\nu= \pm \mu$. Therefore, we have $\boldsymbol{v}=\boldsymbol{\gamma}(s)+\mu \boldsymbol{n}(s) \pm \mu \boldsymbol{e}(s)$.

(3) Since $\bar{h}_{v}^{\prime \prime}(s)=\left\langle-\gamma(s)+\kappa_{g}(s) \boldsymbol{n}(s), \boldsymbol{v}\right\rangle=0$, we have $\mu=-\frac{1}{\delta(\boldsymbol{\gamma}(s)) \kappa_{g}(s)}$, so that

$$
\boldsymbol{v}=\boldsymbol{\gamma}(s)-\frac{1}{\delta(\gamma(s)) \kappa_{g}(s)} \boldsymbol{n}(s) \mp \frac{1}{\delta(\gamma(s)) \kappa_{g}(s)} \boldsymbol{e}(s)
$$

(4) Since $\bar{h}_{v}^{\prime \prime \prime}(s)=\left\langle\left(-1+\kappa_{g}{ }^{2}(s)\right) \boldsymbol{t}(s)+\kappa_{g}{ }^{\prime}(s) \boldsymbol{n}(s)+\kappa_{g}(s) \tau_{g}(s) \boldsymbol{e}(s), \boldsymbol{v}\right\rangle, \frac{\kappa_{g}{ }^{\prime}(s)}{\kappa_{g}(s)} \mp \tau_{g}(s)=0$. It follows that $\sigma(s)=0$.

(5) We have $\bar{h}_{v}^{(4)}(s)=\left\langle\left(1-\kappa_{g}{ }^{2}(s)\right) \gamma(s)+3 \kappa_{g}(s) \kappa_{g}{ }^{\prime}(s) \boldsymbol{t}+\left(-\kappa_{g}(s)+\kappa_{g}{ }^{\prime \prime}(s)+\kappa_{g}{ }^{3}(s)+\right.\right.$ $\left.\left.\kappa_{g}(s) \tau_{g}{ }^{2}(s)\right) \boldsymbol{n}(s)+\left(\kappa_{g}{ }^{\prime}(s) \tau_{g}(s)+\kappa_{g}(s) \tau_{g}{ }^{\prime}(s)\right) \boldsymbol{e}(s), \boldsymbol{v}\right\rangle=0$, so that

$$
\frac{1}{\kappa_{g}(s)}\left(\kappa_{g}^{\prime \prime}(s) \mp \kappa_{g}^{\prime}(s) \tau_{g}(s) \mp \kappa_{g}(s) \tau_{g}^{\prime}(s)\right)=0 .
$$

This is equivalent to the condition that $\sigma^{\prime}(s)=0$.

\section{Singularities of lightlike surfaces}

In this section we study the geometric properties of the lightlike surface of a spacelike curve in de Sitter 3-space. By the propositions in the last section, we can recognize the functions $\kappa_{g}(s)$ and $\sigma(s)$ have special meanings. Here, we consider the case when the lightlike surface has the most degenerate singularities. We have the following proposition.

Proposition 4.1 For a unit speed spacelike curve $\gamma: I \longrightarrow S_{1}^{3}$ with $\left\langle t^{\prime}(s), t^{\prime}(s)\right\rangle \neq 1$, we have the followings:

(1) The singularities of $L S_{\gamma}^{ \pm}$is the set $\left\{(s, u) \mid u=-\frac{1}{\kappa_{g}(s) \delta(\gamma(s))} s \in I\right\}$.

(2) If $L S_{\gamma}^{ \pm}\left(s,-\frac{1}{\kappa_{g}(s) \delta(\boldsymbol{\gamma}(s))}\right)=\boldsymbol{v}_{0}^{ \pm}$is a contact vector, then $\gamma(s) \in L C^{2}\left(\boldsymbol{u}_{0}^{ \pm}\right)$for any $s \in I$ and $\sigma(s) \equiv 0$. Moreover, Image $L S^{ \pm} \subset L C^{2}\left(\boldsymbol{u}_{0}\right)$.

Proof. By straightforward calculations, we have

$$
\begin{aligned}
\frac{\partial L S_{\gamma}^{ \pm}}{\partial s} & =\left(1+u \kappa_{g}(s) \delta(\gamma(s))\right) \boldsymbol{t}(s)+u \tau_{g}(s)(\boldsymbol{e}(s) \pm \boldsymbol{n}(s)) \\
\frac{\partial L S_{\gamma}^{ \pm}}{\partial u} & =\boldsymbol{n}(s) \pm \boldsymbol{e}(s) .
\end{aligned}
$$

The above two vectors are linearly dependent if and only if $1+u \kappa_{g}(s) \delta(\gamma(s))=0$. This completes the proof of the assertion (1).

For a smooth function $\mu: I \longrightarrow \mathbb{R}$, we define a mapping $f_{\mu}: I \longrightarrow \mathbb{R}_{1}^{4}$ by

$$
f_{\mu}(s)=\gamma(s)+\mu(s) \boldsymbol{n}(s) \pm \mu(s) \boldsymbol{e}(s) .
$$


Suppose that $f_{\mu}(s)=\boldsymbol{v}_{0}^{ \pm}$is a constant. Then we have

$$
\begin{aligned}
\frac{d f_{\mu}(s)}{d s} & =\left(-1+\mu(s) \kappa_{g}(s) \delta(\gamma(s))\right) \boldsymbol{t}(s) \\
& +\left(\mu^{\prime}(s) \pm \mu(s) \tau_{g}(s)\right) \boldsymbol{n}(s)+\left(\mu(s) \tau_{g}(s) \pm \mu^{\prime}(s)\right) \boldsymbol{e}(s) \\
& =0
\end{aligned}
$$

Since the singularities are $\mu(s)=-\frac{1}{\kappa_{g}(s) \delta(\gamma(s))}, \mu^{\prime}(s)=\frac{\kappa_{g}{ }^{\prime}(s) \delta(\boldsymbol{\gamma}(s))}{\kappa_{g}{ }^{2}(s)}$. Substituting this relation into the previous equality, we have $\sigma(s)=0$. Under this condition $L S_{\gamma}^{ \pm}\left(s,-\frac{1}{\kappa_{g}(s) \delta(\boldsymbol{\gamma}(s))}\right)=$ $\boldsymbol{\gamma}(s)-\frac{1}{\kappa_{g}(s) \delta(\boldsymbol{\gamma}(s))} \boldsymbol{n}(s) \mp \frac{1}{\kappa_{g}(s) \delta(\boldsymbol{\gamma}(s))} \boldsymbol{e}(s)=\boldsymbol{v}_{0}^{ \pm}$is constant, so that the relation

$$
\left\langle\gamma(s), \boldsymbol{v}_{0}^{ \pm}\right\rangle=\left\langle\gamma(s), \boldsymbol{\gamma}(s)-\frac{1}{\kappa_{g}(s) \delta(\boldsymbol{\gamma}(s))}(\boldsymbol{n}(s) \pm \boldsymbol{e}(s))\right\rangle=1
$$

holds. Moreover, we also have

$$
\left\langle L S_{\gamma}^{ \pm}(s, u), \boldsymbol{v}_{0}^{ \pm}(s)\right\rangle=\left\langle\gamma(s)+u(\boldsymbol{n}(s) \pm \boldsymbol{e}(s)), \boldsymbol{\gamma}(s)-\frac{1}{\kappa_{g}(s) \delta(\boldsymbol{\gamma}(s))}(\boldsymbol{n}(s) \pm \boldsymbol{e}(s))\right\rangle=1
$$

\section{Unfoldings of functions of one-variables}

In this section we use some general results on the singularity theory for families of function germs. Detailed descriptions are found in the book[2]. Let $F:\left(\mathbb{R} \times \mathbb{R}^{r},\left(s_{0}, x_{0}\right)\right) \rightarrow R$ be a function germ. We call $F$ an $r$-parameter unfolding of $f$, where $f(s)=F_{x_{0}}\left(s, x_{0}\right)$. We say that $f$ has $A_{k}$-singularity at $s_{0}$ if $f^{(p)}\left(s_{0}\right)=0$ for all $1 \leq p \leq k$, and $f^{(k+1)}\left(s_{0}\right) \neq 0$. We also say that $f$ has $A_{\geq k}$-singularity at $s_{0}$ if $f^{(p)}\left(s_{0}\right)=0$ for all $1 \leqq p \leqq k$. Let $F$ be an unfolding of $f$ and $f(s)$ has $A_{k}$-singularity $(k \geqq 1)$ at $s_{0}$. We denote the $(k-1)$-jet of the partial derivative $\frac{\partial F}{\partial x_{i}}$ at $s_{0}$ by $j^{(k-1)}\left(\frac{\partial F}{\partial x_{i}}\left(s, x_{0}\right)\right)\left(s_{0}\right)=\sum_{j=1}^{k-1} \alpha_{j i} s^{j}$ for $i=1, \ldots, r$. Then $F$ is called a versal unfolding if the $k \times r$ matrix of coefficients $\left(\alpha_{0 i}, \alpha_{j i}\right)$ has rank $k(k \leqq r)$, where $\alpha_{0 i}=\frac{\partial F}{\partial x_{i}}\left(s_{0}, x_{0}\right)$.

We now introduce an important set concerning the unfolding. The discriminant set of $F$ is the set

$$
\mathfrak{D}_{F}=\left\{x \in \mathbb{R}^{r} \mid \text { there exists } s \text { with } F=\frac{\partial F}{\partial s}=0 \text { at }(s, x)\right\} .
$$

Then we have the following well-known result (cf., [4]).

Theorem 5.1 Let $F:\left(\mathbb{R} \times \mathbb{R}^{r},\left(s_{0}, x_{0}\right)\right) \rightarrow \mathbb{R}$ be an $r$-parameter unfolding of $f(s)$ which has the $A_{k}$ singularity at $s_{0}$. Suppose that $F$ is an versal unfolding.

(a) If $k=1$, then $\mathfrak{D}_{F}$ is locally diffeomorphic to $\{0\} \times \mathbb{R}^{r-1}$.

(b) If $k=2$, then $\mathfrak{D}_{F}$ is locally diffeomorphic to $C \times \mathbb{R}^{r-2}$.

(c) If $k=3$, then $\mathfrak{D}_{F}$ is locally diffeomorphic to $S W \times \mathbb{R}^{r-3}$. We also say that a point $x_{0} \in \mathbb{R}^{r}$ is a fold point of a map germ $f:\left(\mathbb{R}^{r}, x_{0}\right) \longrightarrow\left(\mathbb{R}^{r}, f\left(x_{0}\right)\right)$ if there exist diffeomorphism germs $\phi:\left(\mathbb{R}^{r}, x_{0}\right) \longrightarrow\left(\mathbb{R}^{r}, 0\right)$ and $\psi:\left(\mathbb{R}^{r}, f\left(x_{0}\right)\right) \longrightarrow\left(\mathbb{R}^{r}, 0\right)$ such that $\psi \circ \phi\left(x_{1}, \ldots, x_{r}\right)=$ $\left(x_{1}, \ldots, x_{r-1}, x_{r}^{2}\right)$. 
By Proposition 2.1, the discriminant set of the extended height function $\bar{H}(s, \boldsymbol{v})$ is given as follows:

$$
\mathfrak{D}_{\bar{H}}=\{\boldsymbol{\gamma}(s)+u(\boldsymbol{n}(s) \pm \boldsymbol{e}(s)) \mid s \in I, u \in \mathbb{R}\}
$$

We consider a unit speed spacelike curve $\boldsymbol{\gamma}: I \longrightarrow S_{1}^{3}$ with $\left\langle\boldsymbol{t}^{\prime}(s), \boldsymbol{t}^{\prime}(s)\right\rangle \neq 1$ and the extended height function $\bar{H}$ of $\gamma$. Then we have the following proposition.

Proposition 5.2 Suppose that $\boldsymbol{v} \in \mathfrak{D}_{\bar{H}}$ and $\bar{h}_{v}(s)=\bar{H}(s, \boldsymbol{v})$. If $\bar{h}_{v}$ has $A_{k}$-singularity at $s$ $(k=1,2,3)$, then $\bar{H}$ is a versal unfolding of $\bar{h}_{v}$.

Proof. We denote that

$$
\boldsymbol{\gamma}(s)=\left(x_{1}(s), x_{2}(s), x_{3}(s), x_{4}(s)\right) \text { and } \boldsymbol{v}=\left(v_{1}, v_{2}, v_{3}, \pm \sqrt{1-\nu}\right)
$$

where $\nu=v_{1}^{2}+v_{2}^{2}+v_{3}^{2}$. Under this notation we have

$$
\bar{H}(s, \boldsymbol{v})=-x_{1}(s) v_{1}+x_{2}(s) v_{2}+x_{3}(s) v_{3} \pm x_{4}(s) \sqrt{1-\nu}-1 .
$$

Thus we have

$$
\begin{gathered}
\frac{\partial \bar{H}}{\partial v_{1}}(s, \boldsymbol{v})=-x_{1}(s) \pm \frac{v_{1}}{\sqrt{1-\nu}} x_{4}(s) \\
\frac{\partial \bar{H}}{\partial v_{i}}(s, \boldsymbol{v})=x_{i}(s) \mp \frac{v_{i}}{\sqrt{1-\nu}} x_{4}(s)(i=2,3) .
\end{gathered}
$$

We also have

$$
\begin{gathered}
\frac{\partial}{\partial s} \frac{\partial \bar{H}}{\partial v_{1}}(s, \boldsymbol{v})=-x_{1}{ }^{\prime}(s) \mp \frac{v_{1}}{\sqrt{1-\nu}} x_{4}{ }^{\prime}(s) \\
\frac{\partial}{\partial s} \frac{\partial \bar{H}}{\partial v_{i}}(s, \boldsymbol{v})=x_{i}{ }^{\prime}(s) \pm \frac{v_{i}}{\sqrt{1-\nu}} x_{4}{ }^{\prime}(s)(i=2,3)
\end{gathered}
$$

and

$$
\begin{gathered}
\frac{\partial^{2}}{\partial s^{2}} \frac{\partial \bar{H}}{\partial v_{1}}(s, \boldsymbol{v})=-x_{1}^{\prime \prime}(s) \pm \frac{v_{1}}{\sqrt{1-\nu}} x_{4}^{\prime \prime}(s) \\
\frac{\partial^{2}}{\partial s^{2}} \frac{\partial \bar{H}}{\partial v_{i}}(s, \boldsymbol{v})=x_{i}^{\prime \prime}(s) \mp \frac{v_{i}}{\sqrt{1-\nu}} x_{4}^{\prime \prime}(s)(i=2,3) .
\end{gathered}
$$

THerefore the 2-jet of $\frac{\partial \bar{H}}{\partial v_{i}}(s, \boldsymbol{v})(i=1,2,3)$ at $s_{0}$ is given by

$$
\begin{aligned}
\frac{\partial \bar{H}}{\partial v_{i}}(s, \boldsymbol{v}) & =\frac{\partial \bar{H}}{\partial v_{i}}\left(s_{0}, \boldsymbol{v}\right)+\frac{1}{2} \frac{\partial}{\partial s} \frac{\partial \bar{H}}{\partial v_{i}}\left(s_{0}, \boldsymbol{v}\right)\left(s-s_{0}\right)+\frac{1}{6} \frac{\partial^{2}}{\partial s^{2}} \frac{\partial \bar{H}}{\partial v_{i}}\left(s_{0}, \boldsymbol{v}\right)\left(s-s_{0}\right)^{2} \\
& =\alpha_{0, i}+\frac{1}{2} \alpha_{1, i}\left(s-s_{0}\right)+\frac{1}{6} \alpha_{2, i}\left(s-s_{0}\right)^{2}
\end{aligned}
$$

It is enough to show that the rank of the matrix $A$ is 3 , where

$$
A=\left(\begin{array}{lll}
\alpha_{0,1} & \alpha_{0,2} & \alpha_{0,3} \\
\alpha_{1,1} & \alpha_{1,2} & \alpha_{1,3} \\
\alpha_{2,1} & \alpha_{2,2} & \alpha_{2,3}
\end{array}\right)
$$


We denote that

$$
A(i, j, k)=\operatorname{det}\left(\begin{array}{ccc}
x_{i}(s) & x_{j}(s) & x_{k}(s) \\
x_{i}{ }^{\prime}(s) & x_{j}{ }^{\prime}(s) & x_{k}{ }^{\prime}(s) \\
x_{i}^{\prime \prime}(s) & x_{j}{ }^{\prime \prime}(s) & x_{k}{ }^{\prime \prime}(s)
\end{array}\right)
$$

then we have

$$
\begin{aligned}
& \operatorname{det} A=-\left(A(1,2,3) \mp \frac{v_{1}}{\sqrt{1-\nu}} A(4,2,3) \mp \frac{v_{2}}{\sqrt{1-\nu}} A(1,4,3) \mp \frac{v_{3}}{\sqrt{1-\nu}} A(1,2,4)\right) \\
& = \pm\left\langle\left(v_{1}, v_{2}, v_{3}, \pm \sqrt{1-\nu}\right),(-A(2,3,4),-A(1,3,4), A(1,2,4),-A(1,2,3))\right\rangle \\
& = \pm\left\langle\left(v_{1}, v_{2}, v_{3}, \pm \sqrt{1-\nu}\right),\left|\begin{array}{cccc}
-\boldsymbol{e}_{1} & \boldsymbol{e}_{2} & \boldsymbol{e}_{3} & \boldsymbol{e}_{4} \\
x_{1}(s) & x_{2}(s) & x_{3}(s) & x_{4}(s) \\
x_{1}^{\prime}(s) & x_{2}^{\prime}(s) & x_{3}{ }^{\prime}(s) & x_{4}{ }^{\prime}(s) \\
x_{1}^{\prime \prime}(s) & x_{2}^{\prime \prime}(s) & x_{3}^{\prime \prime}(s) & x_{4}^{\prime \prime}(s)
\end{array}\right|\right\rangle \\
& = \pm \frac{1}{\sqrt{1-\nu}}\left\langle\boldsymbol{v}, \gamma(s) \wedge \gamma^{\prime}(s) \wedge \gamma^{\prime \prime}(s)\right\rangle \text {. }
\end{aligned}
$$

Since $\boldsymbol{v} \in \mathfrak{D}_{\bar{H}}$ is a singular point,

$$
\boldsymbol{v}=\boldsymbol{\gamma}(s)-\frac{1}{\delta(\boldsymbol{\gamma}(s)) \kappa_{g}(s)}(\boldsymbol{n}(s) \pm \boldsymbol{e}(s))
$$

Moreover, we have

$$
\begin{aligned}
\gamma(s) \wedge \gamma^{\prime}(s) \wedge \gamma^{\prime \prime}(s) & =\gamma(s) \wedge \boldsymbol{t}(s) \wedge\left(-\boldsymbol{\gamma}(s)+\kappa_{g}(s) \boldsymbol{n}(s)\right) \\
& =\kappa_{g}(s) \boldsymbol{\gamma}(s) \wedge \boldsymbol{t}(s) \wedge \boldsymbol{n}(s) \\
& =\kappa_{g}(s) \boldsymbol{e}(s)
\end{aligned}
$$

Therefore we have

$$
\begin{aligned}
\operatorname{det} A & = \pm \frac{1}{\sqrt{1-\nu}}\left\langle\boldsymbol{\gamma}(s)-\frac{1}{\delta(\boldsymbol{\gamma}(s)) \kappa_{g}(s)} \boldsymbol{n}(s) \mp \frac{1}{\delta(\boldsymbol{\gamma}(s)) \kappa_{g}(s)} \boldsymbol{e}(s), \kappa_{g}(s) \boldsymbol{e}(s)\right\rangle \\
& =-\frac{\delta(\boldsymbol{\gamma}(s))}{\sqrt{1-\nu}} \neq 0
\end{aligned}
$$

This completes the proof.

Proof of Theorem 2.2. Let $\gamma: I \longrightarrow S_{1}^{3}$ be a unit speed spacelike curve with $\left\langle t^{\prime}(s), t^{\prime}(s)\right\rangle \neq 1$. For $\boldsymbol{v}_{0}^{ \pm}=L S_{\gamma}^{ \pm}\left(s_{0}, u_{0}\right)$, we define a function $\mathcal{H}: S_{1}^{3} \longrightarrow \mathbb{R}$ by $\mathcal{H}(\boldsymbol{u})=\left\langle\boldsymbol{u}, \boldsymbol{v}_{0}^{ \pm}\right\rangle-1$. Then we have $\bar{h}_{v_{0}^{ \pm}}(s)=\mathcal{H}(\boldsymbol{\gamma}(s))$. Since $L C^{2}\left(\boldsymbol{v}_{0}^{ \pm}\right)=\mathcal{H}^{-1}(0)$ and 0 is a regular value of $\mathcal{H}, \bar{h}_{v_{0}^{ \pm}}$has the $A_{k}$-singularity at $s_{0}$ if and only if $\boldsymbol{\gamma}$ and $L C^{2}\left(\boldsymbol{v}_{0}^{ \pm}\right)$have $k+1$-point contact for $s_{0}$. It follows from Proposition 3.1, Theorem 5.1 and Proposition 5.2 that the proof of Theorem 2.2 is completed.

\section{Generic properties of spacelike curves}

In this section we consider generic properties of spacelike curves in $S_{1}^{3}$. The main tool is a kind of transversality theorems. Let $\operatorname{Emb}_{s p}\left(I, S_{1}^{3}\right)$ be the space of spacelike embeddings $\gamma$ : 
$I \longrightarrow S_{1}^{3}$ with $\left\langle\boldsymbol{t}^{\prime}, \boldsymbol{t}^{\prime}\right\rangle \neq 1$ equipped with Whitney $C^{\infty}$-topology. We also consider the function $\mathcal{H}: S_{1}^{3} \times S_{1}^{3} \longrightarrow \mathbb{R}$ defined by $\mathcal{H}(\boldsymbol{u}, \boldsymbol{v})=\langle\boldsymbol{u}, \boldsymbol{v}\rangle-1$. We claim that $\mathcal{H}_{v}$ is a submersion for any $\boldsymbol{v} \in S_{1}^{3}$, where $\mathcal{H}_{v}(\boldsymbol{u})=\mathcal{H}(\boldsymbol{u}, \boldsymbol{v})$. For any $\boldsymbol{\gamma} \in \operatorname{Emb}_{s p}\left(I, S_{1}^{3}\right)$, we have $\bar{H}=\mathcal{H} \circ\left(\boldsymbol{\gamma} \times i d_{S_{1}^{3}}\right)$. We also have the $\ell$-jet extension

$$
j_{1}^{\ell} \bar{H}: I \times S_{1}^{3} \longrightarrow J^{\ell}(I, \mathbb{R})
$$

defined by $j_{1}^{\ell} \bar{H}(s, \boldsymbol{v})=j^{\ell} \bar{h}_{v}(s)$. We consider the trivialisation $J^{\ell}(I, \mathbb{R}) \equiv I \times \mathbb{R} \times J^{\ell}(1,1)$. For any submanifold $Q \subset J^{\ell}(1,1)$, we denote that $\widetilde{Q}=I \times\{0\} \times Q$. Then we have the following proposition as a corollary of Lemma 6 in Wassermann[9].

Proposition 6.1 Let $Q$ be a submanifold of $J^{\ell}(1,1)$. Then the set

$$
T_{Q}=\left\{\boldsymbol{\gamma} \in \operatorname{Emb}_{s p}\left(I, S_{1}^{3}\right) \mid j_{1}^{\ell} \bar{H} \text { is transversal to } \widetilde{Q}\right\}
$$

is a residual subset of $\operatorname{Emb}_{s p}\left(I, S_{1}^{3}\right)$. If $Q$ is a closed subset, then $T_{Q}$ is open.

Let $f:(\mathbb{R}, 0) \longrightarrow(\mathbb{R}, 0)$ be a function germ which has an $A_{k}$-singularity at 0 . It is wellknown that there exists a diffeomorphism germ $\phi:(\mathbb{R}, 0) \longrightarrow(\mathbb{R}, 0)$ such that $f \circ \phi(s)= \pm s^{k+1}$. This is the classification of $A_{k}$-singularities. For any $z=j^{\ell} f(0) \in J^{\ell}(1,1)$, we have the orbit $L^{\ell}(z)$ given by the action of the Lie group of $\ell$-jets of diffeomorphism germs. If $f$ has an $A_{k^{-}}$ singularity, then the codimension of the orbit is $k$. There is another characterisation of versal unfoldings as follows(cf., [6]):

Proposition 6.2 Let $F:\left(\mathbb{R} \times \mathbb{R}^{r}, 0\right) \longrightarrow(\mathbb{R}, 0)$ be an $r$-parameter unfolding of $f:(\mathbb{R}, 0) \longrightarrow$ $(\mathbb{R}, 0)$ which has an $A_{k}$-singularity at 0 . Then $F$ is a versal unfolding if and only if $j_{1}^{\ell} F$ is transversal to the orbit $\widehat{L^{\ell}\left(j^{\ell} f(0)\right)}$ for $\ell \geq k+1$.

Here, $j_{1}^{\ell} F:\left(\mathbb{R} \times \mathbb{R}^{r}, 0\right) \longrightarrow J^{\ell}(\mathbb{R}, \mathbb{R})$ is the $\ell$-jet extension of $F$ given by $j_{1}^{\ell} F(s, x)=j^{\ell} F_{x}(s)$.

The generic classification theorem is given as follows:

Theorem 6.3 There exists an open and dense subset $\mathcal{O} \subset \operatorname{Emb}_{s p}\left(I, S_{1}^{3}\right)$ such that for any $\gamma \in \mathcal{O}$, the lightlike surface $L S_{\gamma}^{ \pm}$of $\boldsymbol{\gamma}$ is locally diffeomorphic to the cuspidal edge or the swallowtail at any singular point.

Proof. For $\ell \geq 4$, we consider the decomposition of the jet space $J^{\ell}(1,1)$ into $L^{\ell}(1)$ orbits. We now define a semi-algebraic set by

$$
\Sigma^{\ell}=\left\{z=j^{\ell} f(0) \in J^{\ell}(1,1) \mid f \text { has an } A_{\geq 4} \text {-singularity }\right\} .
$$

Then the codimension of $\Sigma^{\ell}$ is 4 . Therefore, the codimension of $\widetilde{\Sigma_{0}}=I \times\{0\} \times \Sigma^{\ell}$ is 5 . We have the orbit decomposition of $J^{\ell}(1,1)-\Sigma^{\ell}$ into

$$
J^{\ell}(1,1)-\Sigma^{\ell}=L_{0}^{\ell} \cup L_{1}^{\ell} \cup L_{2}^{\ell} \cup L_{3}^{\ell},
$$

where $L_{k}^{\ell}$ is the orbit through an $A_{k}$-singularity. Thus, the codimension of $\widetilde{L_{k}^{\ell}}$ is $k+1$. We consider the $\ell$-jet extension $j_{1}^{\ell} \bar{H}$ of the extended spacelike height function $\bar{H}$. By Proposition 6.1 , there exists an open and dense subset $\mathcal{O} \subset \operatorname{Emb}\left(I, S_{1}^{3}\right)$ such that $j_{1}^{\ell} \bar{H}$ is transversal to $\widetilde{L_{k}^{\ell}}$ $(k=0,1,2,3)$ and the orbit decomposition of $\widetilde{\Sigma^{\ell}}$. This means that $j_{1}^{\ell} \bar{H}\left(I \times S_{1}^{3}\right) \cap \widetilde{\Sigma^{\ell}}=\emptyset$ and $\bar{H}$ is a versal unfolding of $h$ at any point $\left(s_{0}, v_{0}\right)$. By Theorem 5.1, the discriminant set of $\bar{H}$ (i.e., the lightlike surface of $\gamma$ ) is locally diffeomorphic to the cuspidal edge or the swallow tail if the point is singular. 


\section{References}

[1] M.A.Akivis and V.V.Goldberg, Differential Geometry of Varieties with Degenerate Gauss Maps, Canadian Mathematical Society, Springer, 2004.

[2] J. W. Bruce and P. J. Giblin, Curves and singularities (second edition). Cambridge University press (1992)

[3] S. Chandrasekhar, The Mathematical Theory of Black Holes, International Series of Monographs on Physics. 69 Oxford Univeristy press, 1983.

[4] S.Izumiya, D.Pei and T.Sano, The lightcone Gauss map and the lightcone developable of a spacelike curve in Minkowski 3-space, Glasgow Math. J. 42 (2000), 75-89.

[5] S. Izumiya, D-H. Pei and T. Sano, Horospherical surfaces of curves in Hyperbolic space, Publ. Math. (Debrecen) 64 (2004),1-13

[6] J. Martinet, Singularities of Smooth Functions and Maps, London Math. Soc. Lecture Note Series, Cambridge Univ. Press, 58 (1982)

[7] C. W. Misner, K. S. Thorpe and J. W. Wheeler, Gravitation, W. H. Freeman and Co., San Francisco, CA, 1973.

[8] B. O'neil, Semi-Riemannian Geometry. Academic Press, New York (1983).

[9] G. Wassermann, Stability of Caustics. Math. Ann., 210 (1975), 43-50

Takesi Fusho, Department of Mathematics, Hokkaido University, Sapporo 060-0810,Japan Shyuichi Izumiya, Department of Mathematics, Hokkaido University, Sapporo 060-0810,Japan e-mail:izumiya@math.sci.hokudai.ac.jp 